\title{
Processing of Graphene combining Optical Detection and Scanning Probe Lithography
}

\author{
Sören Zimmermann ${ }^{1}$, a , Alexander van Düllen ${ }^{1}$, Markus Wieghaus ${ }^{1}$, and Sergej Fatikow ${ }^{1}$ \\ ${ }^{1}$ Division Microrobotics and Control Engineering, University of Oldenburg, D-26129 Oldenburg, Germany
}

\begin{abstract}
This paper presents an experimental setup tailored for robotic processing of graphene with in-situ vision based control. A robust graphene detection approach is presented applying multiple image processing operations of the visual feedback provided by a high-resolution light microscope. Detected graphene flakes can be modified using a scanning probe based lithographical process that is directly linked to the in-situ optical images. The results of this process are discussed with respect to further application scenarios.
\end{abstract}

\section{Introduction}

Graphene, an atomically thin sheet consisting of $\mathrm{sp}^{2}$ hybridized carbon atoms, has gained a lot of attention from the scientific community within the last decade. Particularly the excellent physical properties such as high carrier mobility [1], mechanical stiffness [2] as well as good environmental stability promise multiple application perspectives of graphene, e.g. in sensors [3], next-generation electronics [4], nanoelectromechanical systems [5] and optoelectronics [6, 7].

One of the main advantages of graphene, compared to other nanomaterials like nanowires, is its twodimensional structure making it compatible with the wellknown lithographical techniques developed for silicon.

However, most of these techniques apply polymer based resists that may leave a substantial amount of polymer residues on the surface. These residues are in turn going along with considerable deteriorations of the excellent and desired properties of graphene [8]. Hence, the development of resist-free lithographical processes is of significant importance for realizing high-performance graphene-based devices.

One promising approach for resist-free lithography on graphene is based on the scanning probe principle $[9,10$, $11,12]$. These techniques use precise robotic setups to actuate a miniaturized probe in order to cut, oxidize or etch graphene with high spatial resolution. So far, these techniques are still at an early development stage and thus, further research is mandatory to push them closer to application maturity.

This work presents a tailored experimental setup allowing to combine high-resolution optical imaging with robotic nanomanipulation. In this way, vision-based information can be applied directly to identify and classify graphene and to define a target area that is subsequently processed by scanning probe lithography. Within the next section, the overall experimental setup is briefly introduced. Afterwards, the optical identification of single- and bilayer graphene is described and evaluated in detail. Next, the application of scanning probe lithography on a predefined target area will be presented and validated. Finally, the results are summarized and an outlook targeting on further developments is given.

\section{Experimental Setup}

The experimental setup is illustrated in Figure 1. Overall, the setup consists of a high-resolution light microscope as well as of two individual robotic stages. Here, one stage is carrying the graphene samples while the other stage actuates the probe used for the lithographical process (Figure 1).

The light microscope is equipped with an infinity corrected objective (Mitutoyo) providing a spatial resolution of $500 \mathrm{~nm}$ and offering an extraordinary large working distance of $15 \mathrm{~mm}$. In this way, sufficient space between target sample and microscope remains, allowing to align the robotic driven probe in between.

The sample stage consist of three linear axes (SmarAct SLC). Each axis is slip-stick driven and equipped with internal positioning sensors providing a stroke of $30 \mathrm{~mm}$ and a closed-loop accuracy of approximately $20 \mathrm{~nm}$ in each spatial direction.

The probe intended for lithographical processing is mounted onto a piezo-based fine positioning system (Physik Instrumente MARS) that is equipped with capacitive position sensors. This fine positioning system offers a travel range of $300 \mu \mathrm{m}$ in each spatial direction with a closed-loop accuracy of approximately $1 \mathrm{~nm}$.

\footnotetext{
${ }^{\mathrm{a}}$ Corresponding author: soeren.zimmermann@uni-oldenburg.de
} 


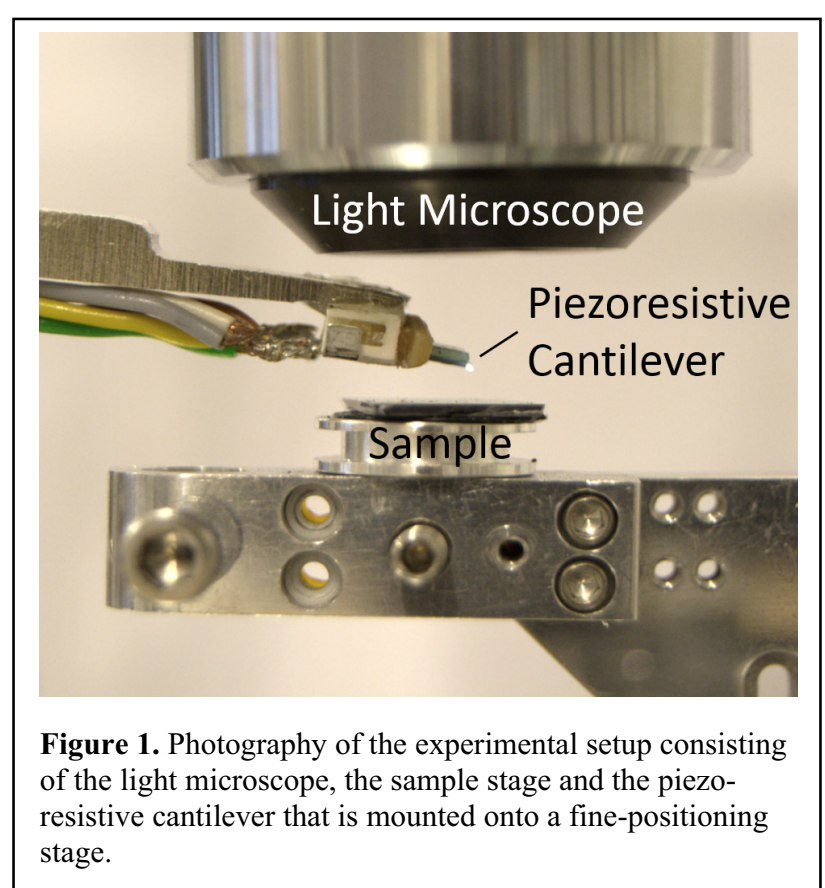

Within this work, the used probe consist of a piezoresistive cantilever (SEIKO PRC 400) tailored for contact atomic force microscopy (AFM). This probe utilizes a Wheatstone bridge circuit for detecting applied normal forces to the cantilever.

More detailed information on this probe will be given within chapter 4. Further information on the used components as well as other application scenarios of this robotic platform can be found within our previous work [13]. For control and processing of the multiple sensor information, a special tailored software framework has been applied that has already been described extensively elsewhere $[14,15,16]$.

\section{Optical Detection of Graphene}

For fabrication of graphene samples, mechanical exfoliation of graphite using adhesive tape has been applied [17]. Here, silicon samples with a well-defined oxide layer (300 nm oxide thickness) have been used as the substrate. This substrate allows for detection of graphene using conventional light microscopes due to a strong amplitude modulation of reflected light at the interface of graphene, silicon dioxide and silicon [18]. This amplitude modulation causes a marginal but significant colour shift for graphene and allows to distinguish between single- and multi-layer graphene areas $[19,20]$. However, inhomogeneous illumination of the substrate causes slightly different colour shifts that depend on the location within the light microscope image. Hence, reliable detection and classification of graphene using light microscopes require different image processing steps. Within this work the OpenCV image processing library [21] has been used for all standard image processing operations. Furthermore, tailored image processing filters have been designed and will be described in more detail.

The first image processing step for graphene detection is the nonuniform lighting elimination approach proposed

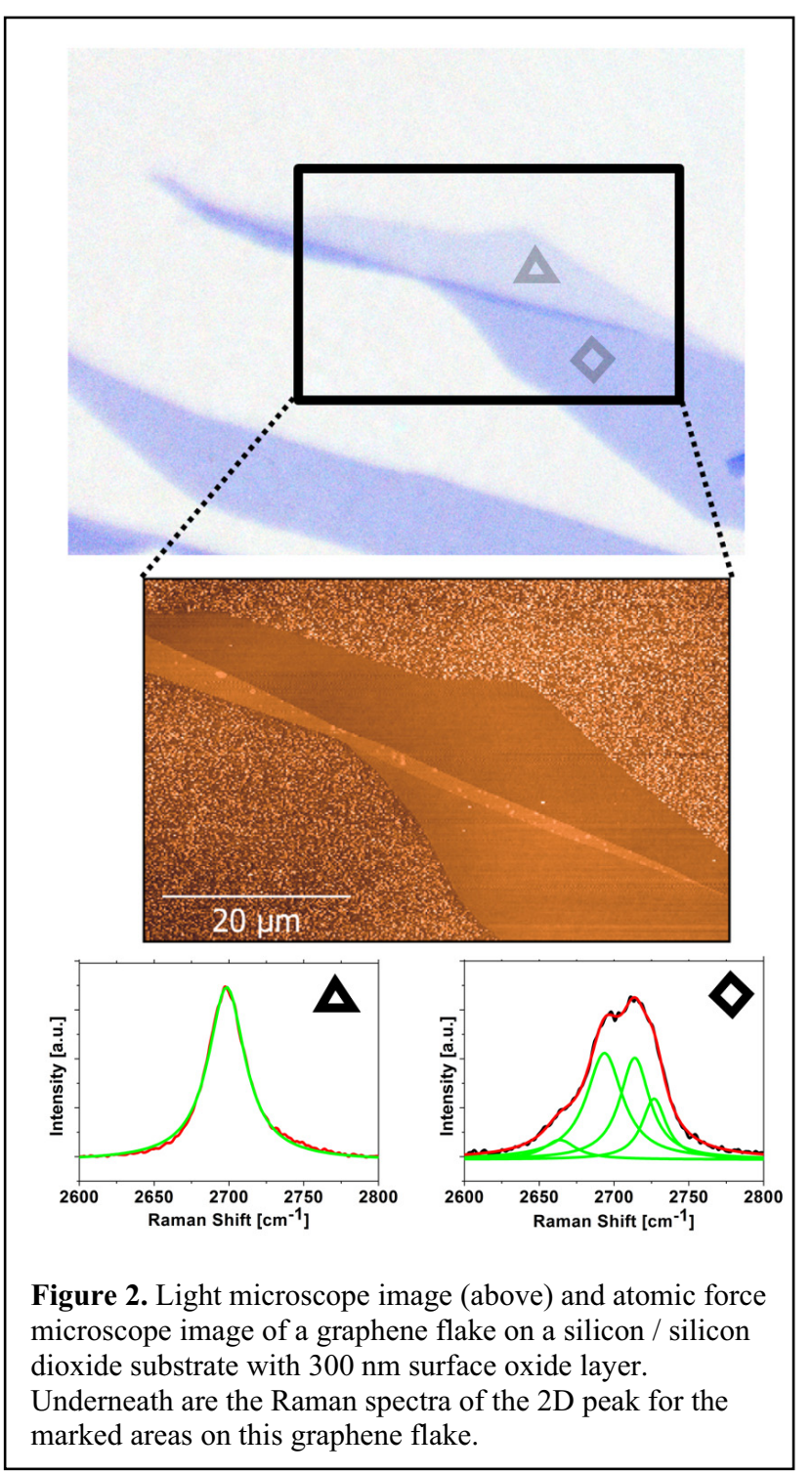

by Nolen et. al. [20]. This approach uses a clean image of the pure substrate (background) to substract the inhomogeneous light intensity. Afterwards, contrast enhancement has been applied to pronounce the colour shift between graphene and background. A typical light microscope image of a graphene flake on the pure substrate after these image processing steps is shown in Figure 2 (top). Here, different areas with different graphene thicknesses can be differentiated. For further classification of this graphene sample, AFM as well as Raman measurements have been performed. The results are summarized in Figure 2. The AFM image has been acquired in intermittent contact mode using a JPK NanoWizard II setup. The image reveals that the graphene flake is extremely smooth and homogeneous except for a thick narrow stripe between the two larger areas. The two homogeneous areas have been analysed by means of Raman spectroscopy using an excitation wavelength of $514 \mathrm{~nm}$ and a beam power of $2 \mathrm{~mW}$ at the spots indicated in Figure 2. Overall, the Raman spectrum of graphene can provide multiple information on the physical properties [22]. Particularly, the so-called 2D peak occurring typically at a Raman Shift around $2700 \mathrm{~cm}^{-1}$ captures the electronic structure of graphene 
and is due to a double resonance Raman scattering process [22]. As the electronic structure of graphene evolves with the number of layers, the $2 \mathrm{D}$ peak represents a well-defined fingerprint for single- and bi-layer graphene. The spectra shown in Figure 2 reveal two completely different $2 \mathrm{D}$ peak shapes. The left peak is highly symmetric and can be fitted adequately by a single lorentzian curve. In contrast, the right $2 \mathrm{D}$ peak splits up into four sub-components. Considering the explanation given in [22], the symmetric peak corresponds to singlelayer graphene while the peak with the four subcomponents corresponds to bi-layer graphene on a silicon/ silicon dioxide substrate with $300 \mathrm{~nm}$ oxide layer. Based on the classification described above, this graphene flake can be used for calibration measurements in order to design a suitable image filter for graphene detection and classification. Therefore, the RGB values of the preprocessed image (nonuniform lighting elimination, contrast enhancement) have been recorded within a single-pixel that corresponds either to the background, single- or bi-layer graphene, respectively. While the distribution of the $B$ channel values is comparable for all cases, the results for the $R$ and $G$ values are illustrated in Figure 3. Here, it can be clearly seen that the $R$ and $G$ values show significant downshifts for both single- and bi-layer graphene.

This information has been used for creating a suitable image filter for graphene detection. This filter differs from previous graphene detection approaches [20]. Overall, the filter converts the RGB image into a greyscale image using an RGB selective bandpass filter for different graphene thicknesses. This filter has been created using a simple weighting function (here: gauss function) based on the contribution of $R$ and $G$ shown in Figure 3.

In this way, only a small band of $\mathrm{R}$ and $\mathrm{G}$ values can contribute to a greyscale image while all other values are set to a greyscale value of zero. For example, for detecting single-layer graphene only $\mathrm{R}$ values between 67 and 71 can contribute, while $R$ values of 69 are weighted higher than $\mathrm{R}$ values of 67 or 71 (Figure 3).

For object detection, the created greyscale image is thresholded and converted into a binary image. Examples of the binary images applying filters tailored for singleand bi-layer graphene detection are shown in Figure 4.

Here, it can be clearly seen that the filters show an
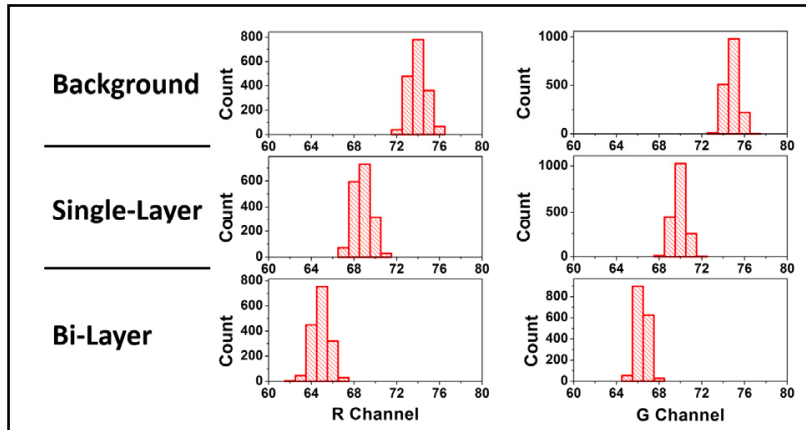

Figure 3. Distribution of the $\mathrm{R}$ and $\mathrm{G}$ Values of the light microscope camera measured within one pixel of the background, the single-layer graphene as well as the bilayer graphene area.

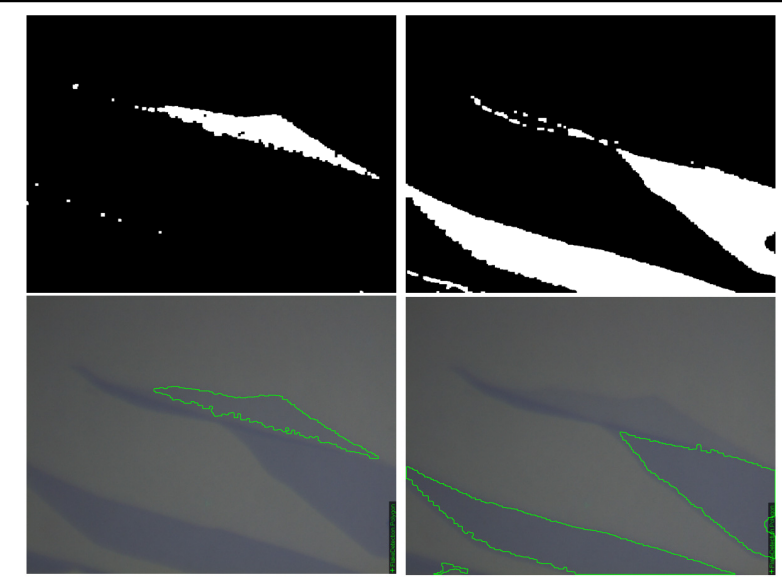

Figure 4. Binary images of the light microscope after RGB selective filtering targeting on single- (top left) and bi-layer (top right) graphene. The results of the BLOB detection for each case are shown underneath.

excellent performance for both single- and bi-layer graphene detection. Solely minor parts at the edges of the graphene flakes are assigned incorrectly. However, this is due to the limited resolution of the light microscope leading to a mixture of graphene and background within a single pixel. A suitable approach for eliminating the incorrectly assigned edges is a binary morphology filter.

In order to detect a single graphene flake, a binary large object detection (BLOB) algorithm has been applied that is provided by OpenCV. The results of the BLOB detection are illustrated in Figure 4, revealing an excellent performance.

Additionally, the developed graphene detection approach has been tested applying a continuous video stream of the microscope camera while scanning a macroscopic sample using the robotic driven sample stage described in chapter 2. Here, all found graphene flakes have been assigned correctly. Therefore, the herein presented optical graphene detection approach is robust and suitable for graphene detection and classification.

\section{Probe based Processing of Graphene}

Commonly, graphene flakes fabricated by mechanical exfoliation of graphite cannot be controlled in shape and size. Therefore, graphene flakes need to be tailored using lithographical processes in order to integrate the graphene into a final device. Here, mainly electron beam lithography is applied followed by reactive ion etching techniques [17]. However, due to the need of electron beam sensitive resists like PMMA, the graphene remains contaminated after the process. As every atom of graphene is on its surface, contaminations have a significant influence on the overall physical and chemical properties of graphene [8].

Scanning probe lithography techniques allow for resistfree processing of graphene and thus, these techniques are promising in enabling high-quality graphene devices. Within this work, we present, for the first time, a scanning probe process that is directly linked to optical imaging applying the setup shown in Figure 1. 
So far, different working principles for scanning probe lithography have been proposed, including local anodic oxidation, dip-pen nanolithography and mechanical scratching [9]. For a proof-of-concept study, the latter one has been chosen as it can be applied using a simple unmodified AFM probe. Due to the working principle, this technique is also known as AFM-PlowLithography [11].

A scanning electron micrograph of the herein used AFM probe is shown in Figure 5. This probe is equipped with a silicon nitride tip with a tip radius below $20 \mathrm{~nm}$.

For cutting of graphene using AFM cantilever the applied normal force is a decisive factor $[11,12]$. In order to identify appropriate values for this setup, different line scans have been performed on a bi-layer graphene using multiple normal forces as indicated in Figure 5. Here, the cantilever is scanned with a constant velocity of $4 \mu \mathrm{m} / \mathrm{s}$ across the substrate.

Overall, the applied normal force is directly proportional to the voltage output of the piezoresistive cantilever and can be estimated using the spring constant provided by the manufacturer $(2-4 \mathrm{~N} / \mathrm{m})$ [23]. The values in Figure 5 are calculated appraising a spring constant of $3 \mathrm{~N} / \mathrm{m}$. Therefore, these values should be regarded as an estimation rather than an exact determination.

During the line scans, the voltage output coming from the cantilever has been set to a constant value during lateral scanning using a PI-Loop control of the $\mathrm{z}$-axis.

The AFM scans on the edge of the processed graphene flake reveal that a normal force of approximately $19 \mu \mathrm{N}$ is still insufficient to cut the bi-layer graphene flake. Vice-versa, normal forces above approximately $40 \mu \mathrm{N}$ allow for consistent cutting of graphene. It is also obvious that the graphene tends to flip onto the overall flake at the cutting edges due to the strong adhesive forces on that scale.

Based on this preliminary experiment, a bi-layer graphene flake has been detected by means of image processing as described in chapter 3. Subsequently, this flake has been processed by AFM-Plow-Lithography using a fully-automated process. For this process, the vision based position information coming from the light microscope need to be linked to the position sensor information of the robotic fine positioning stage. Therefore, two calibration procedures are mandatory. On the one hand, image pixel coordinates need to be converted into position sensor data using triangulation. On the other hand, the position of the tip of the cantilever needs to be detected within a top-view light microscope image in order to determine the exact location of the interaction between cantilever and substrate. Here, it is important that the calibration is conducted with the same applied force to the cantilever as the lithographical process itself. Otherwise, the determined position of the location of the interaction would differ significantly due to the self-bending of the cantilever itself. Detailed information on both calibration procedures can be found in our previous work [15].

After the calibration, a target area can be defined within the light microscope image that is processed by a series of AFM line scans with predefined parameters. Here, approximately $40 \mu \mathrm{N}$ normal force has been applied. The
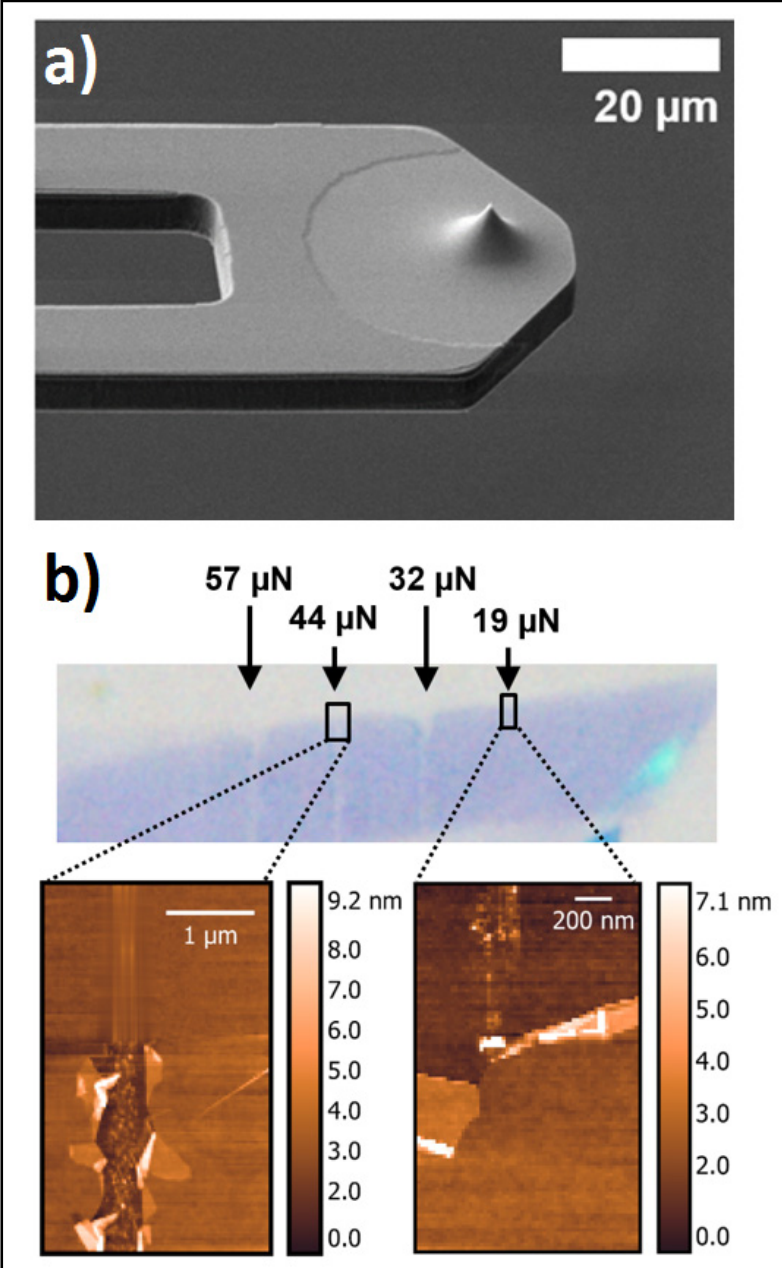

Figure 5. a) - Scanning electron micrograph of the used piezoresistive cantilever and b) - light microscope image of a bi-layer graphene flake that has been modified by AFMplow lithography using different normal forces. Selected areas have been further analysed using AFM imaging as indicated.

scanning velocity of the cantilever was set to $4 \mu \mathrm{m} / \mathrm{s}$ and the lateral distance between two line scans has been set to $400 \mathrm{~nm}$. Due to the limited depth of field of highresolution light microscopes either the cantilever or the sample can be seen in focus. The two rectangular boxes within the light microscope image in Figure 6 (left) define the target area. The larger box defines the area where graphene should be removed while the smaller box defines an area where graphene should remain intact.

An AFM image of the graphene flake after the lithographical process is shown in Figure 6 (right). Principally, the lithographical process has been successful. However, residuals of the scratched graphene remain at the edges and one part of the graphene rectangle is still connected to the initial flake at the right edge. Hence, not all graphene could be removed successfully. Furthermore, the edges are not straight. This is mainly due to the fact that graphene preferentially breaks along predefined axes that are determined by the lattice structure of graphene itself. Nevertheless, except of the edges, the graphene remains entirely uncontaminated. 


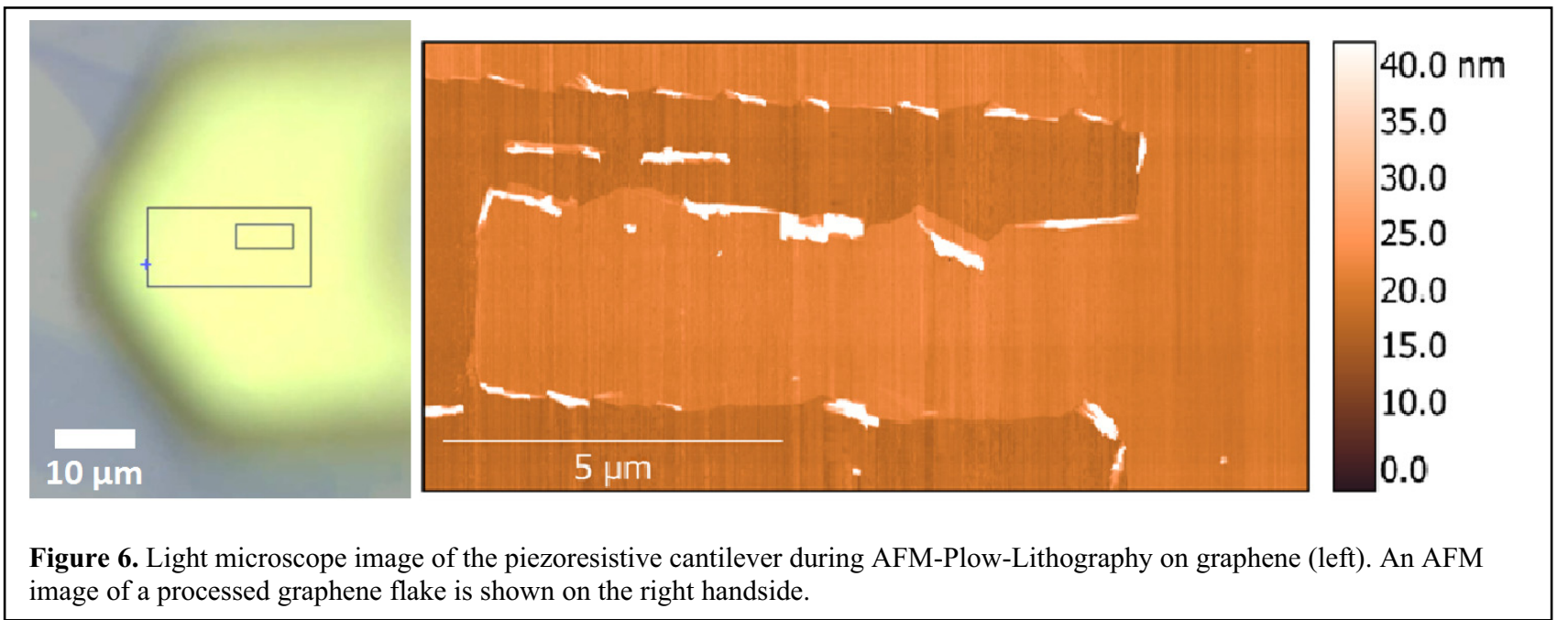

\section{Summary and Outlook}

An experimental setup tailored for automated robotic processing of graphene has been presented. The used light microscope can be applied to detect and classify individual graphene flakes. After detection, the graphene flakes can be processed automatically by combining vision based information with scanning probe lithography.

Overall the combination of a high-resolution light microscope and a scanning probe approach is highly promising for fabrication of high-quality graphene devices. However, the herein applied AFM-PlowLithography is not suitable for accurately shaped graphene samples as graphene residuals and undefined edge structures occur. Therefore, future work will focus on combining optical detection with different probe based lithography principles such as local anodic oxidation.

\section{References}

[1] K. Bolotin, K. Sikes, Z. Jiang, M. Klima, G. Fudenberg, J. Hone, P. Kim, and H. Stormer, "Ultrahigh electron mobility in suspended graphene," Solid State Communications, vol. 146, no. 9-10, pp. 351-355, Jun. 2008.

[2] C. Lee, X. Wei, J. W. Kysar, and J. Hone, "Measurement of the elastic properties and intrinsic strength of monolayer graphene," Science, vol. 321, no. 5887, pp. 385-388, 2008.

[3] K. Kostarelos and K. S. Novoselov, "Graphene devices for life," Nat Nano, vol. 9, no. 10, pp. 744745, Oct. 2014.

[4] F. Schwierz, "Graphene transistors," Nat Nano, vol. 5, no. 7, pp. 487-496, Jul. 2010.

[5] J. S. Bunch, A. M. van der Zande, S. S. Verbridge, I. W. Frank, D. M. Tanenbaum, J. M. Parpia, H. G. Craighead, and P. L. McEuen, "Electromechanical resonators from graphene sheets," Science, vol. 315, no. 5811, pp. 490-493, 2007.

[6] F. Rana, "Graphene optoelectronics: Plasmons get tuned up," Nat Nano, vol. 6, no. 10, pp. 611-612, Oct. 2011.
[7] F. Bonaccorso, Z. Sun, T. Hasan, and A. C. Ferrari, "Graphene photonics and optoelectronics," Nat Photon, vol. 4, no. 9, pp. 611-622, Sep. 2010.

[8] A. Pirkle, J. Chan, A. Venugopal, D. Hinojos, C. W. Magnuson, S. McDonnell, L. Colombo, E. M. Vogel, R. S. Ruoff, and R. M. Wallace, "The effect of chemical residues on the physical and electrical properties of chemical vapor deposited graphene transferred to sio2," Applied Physics Letters, vol. 99, no. 12, p. $122108,2011$.

[9] N. Kurra, R. G. Reifenberger, and G. U. Kulkarni, "Nanocarbon-scanning probe microscopy synergy: Fundamental aspects to nanoscale devices," $A C S$ Appl. Mater. Interfaces, vol. 6, no. 9, pp. 6147-6163, May 2014.

[10]A. Giesbers, U. Zeitler, S. Neubeck, F. Freitag, K. Novoselov, and J. Maan, "Nanolithography and manipulation of graphene using an atomic force microscope," Solid State Communications, vol. 147, no. 9-10, pp. 366-369, Sep. 2008.

[11]B. Vasic, M. Kratzer, A. Matkovic, A. Nevosad, U. Ralevic, D. Jovanovic, C. Ganser, C. Teichert, and R. Gajic, "Atomic force microscopy based manipulation of graphene using dynamic plowing lithography," Nanotechnology, vol. 24, no. 1, p. 015303, 2013.

[12] Y. Zhang, L. Liu, N. Xi, Y. Wang, and Z. Dong, "Cutting graphene using an atomic force microscope based nanorobot," in Nanotechnology (IEEE-NANO), 2010 10th IEEE Conference on, 2010, pp. 639-644.

[13] S. Zimmermann, S. Barragan, and S. Fatikow, "Nanorobotic processing of graphene: A platform tailored for rapid prototyping of graphene-based devices." Nanotechnology Magazine, IEEE, vol. 8, no. 3, pp. 14-19, 2014.

[14]C. Diederichs, M. Bartenwerfer, M. Mikczinski, S. Zimmermann, T. Tiemerding, C. Geldmann, H. Nguyen, C. Dahmen, and S. Fatikow, "A rapid automation framework for applications on the microand nanoscale," in Proceedings of Australasian Conference on Robotics and Automation, 2013.

[15] S. Zimmermann, T. Tiemerding, T. Li, W. Wang, Y. Wang, and S. Fatikow, "Automated mechanical characterization of 2-d materials using sem based 
visual servoing," International Journal of Optomechatronics, vol. 7, no. 4, pp. 283-295, Oct. 2013.

[16] S. Zimmermann, T. Tiemerding, and S. Fatikow, "Automated robotic manipulation of individual colloidal particles using vision-based control," Mechatronics, IEEE/ASME Transactions on, vol. PP, no. 99, pp. 1-8, 2014.

[17] K. S. Novoselov, A. K. Geim, S. V. Morozov, D. Jiang, Y. Zhang, S. V. Dubonos, I. V. Grigorieva, and A. A. Firsov, "Electric field effect in atomically thin carbon films," Science, vol. 306, no. 5696, pp. 666-669, 2004.

[18] P. Blake, E. W. Hill, A. H. Castro Neto, K. S. Novoselov, D. Jiang, R. Yang, T. J. Booth, and A. K. Geim, "Making graphene visible," Applied Physics Letters, vol. 91, no. 6, p. 063124, 2007.

[19]H. Li， J. Wu， X. Huang， G. Lu， J. Yang， X. Lu, Q. Xiong, and H. Zhang, "Rapid and reliable thickness identification of two-dimensional nanosheets using optical microscopy," ACS Nano, vol. 7, no. 11, pp. 10344-10353, Nov. 2013.

[20]C. M. Nolen, G. Denina, D. Teweldebrhan, B. Bhanu, and A. A. Balandin, "High-throughput large-area automated identification and quality control of graphene and few-layer graphene films," ACS Nano, vol. 5, no. 2, pp. 914-922, Feb. 2011.

[21] G. Bradski, "The open cv library," Dr. Dobb's Journal of Software Tools, 2000.

[22] A. C. Ferrari, J. C. Meyer, V. Scardaci, C. Casiraghi, M. Lazzeri, F. Mauri, S. Piscanec, D. Jiang, K. S. Novoselov, S. Roth, and A. K. Geim, "Raman spectrum of graphene and graphene layers," Phys. Rev. Lett., vol. 97, no. 18, pp. 187401-, Oct. 2006.

[23] S. B. Aksu and J. A. Turner, "Calibration of atomic force microscope cantilevers using piezolevers," Review of Scientific Instruments, vol. 78, no. 4, p. 043704, 2007. 